\title{
Targeting Selectins Mediated Biological Activities With Multivalent Probes
}

\author{
Deepak Ganesh, Prashant Jain, Chethan Devanur Shanthamurthy, Suraj Toraskar and \\ Raghavendra Kikkeri *
}

Indian Institute of Science Education and Research, Pune, India

Selectins are type-I transmembrane glycoproteins that are ubiquitously expressed on activated platelets, endothelial cells, and leukocytes. They bind to cell surface glycoproteins and extracellular matrix ligands, regulate the rolling of leukocytes in the blood capillaries, and recruit them to inflammatory sites. Hence, they are potential markers for the early detection and inhibition of inflammatory diseases, thrombosis, cardiovascular disorders, and tumor metastasis. Fucosylated and sialylated glycans, such as sialyl Lewisx, its isoform sialyl Lewisa, and heparan sulfate, are primary selectin ligands. Functionalization of these selectin-binding ligands on multivalent probes, such as

OPEN ACCESS

Edited by:

Zhongjun Li,

Peking University, China

Reviewed by:

Bei Liu,

University of North Carolina at Chapel

Hill, United States

Qinzhe Wang,

University of Utah, United States

*Correspondence:

Raghavendra Kikkeri rkikkeri@iiserpune.ac.in

Specialty section:

This article was submitted to

Chemical Biology,

a section of the journal

Frontiers in Chemistry

Received: 09 September 2021

Accepted: 25 October 2021

Published: 03 December 2021

Citation:

Ganesh D, Jain $P$, Shanthamurthy $C D$,

Toraskar S and Kikkeri R (2021)

Targeting Selectins Mediated

Biological Activities With

Multivalent Probes.

Front. Chem. 9:773027.

doi: 10.3389/fchem.2021.773027 nanoparticles, liposomes, and polymers, not only inhibits selectin-mediated biological activity but is also involved in direct imaging of the inflammation site. This review briefly summarizes the selectin-mediated various diseases such as thrombosis, cancer and recent progress in the different types of multivalent probes used to target selectins.

Keywords: selectins, cancer, nanoparticles, imaging, drug delivery

\section{BACKGROUND}

The first report of blood cells rolling, adhesion and migration on activated endothelial cell surfaces under shear flow conditions came over a decade ago (Chiara et al., 1999). Later, Giulio et al. reported platelet adhesion on damaged vascular cell walls, resulting in secondary phenomena like blood clotting (De Gaetano et al., 2003). However, the exact mechanism and the molecular level details were unknown until Wagner et al. identified the cell adhesion molecule receptor called selectin on the surface of activated endothelial cells (Wagner et al., 1982). Later, it was quantitatively proved that the selectin family of adhesion molecules plays a significant role in this interaction, particularly in leukocyte recruitment to the site of infection or inflammation and platelet-endothelial cell interaction and aggregation (Pachynski et al., 1998). Leukocytes migrate from capillaries via an adhesion cascade which could be classified into three steps: rolling and capture, mediated by selectins, followed by activation and arrest; which is mediated by chemokines and integrins respectively, and arrest, which is mediated by integrins, progressing to transcellular migration. Selectin-triggered endothelial activation leads to leukocyte-assisted tumor cell extravasation and cytokines produced by the tumor cells lead to endothelial activation and leaky vasculature, promoting leukocyte recruitment. Platelets binding to endothelium and tumor cells, promote tumor cell adhesion and this interaction is largely contributed by P-selectin and endothelial activation also triggering leukocyte-assisted tumor cell extravasation. P-Selectin glycoprotein ligand-1 (PSGL-1) and selectin binding triggers intracellular signalling in leukocytes resulting in $\mathrm{NF \kappa B}, \mathrm{MAPK}$, and SRC pathways leading to activation of integrins and secretion of cytokines like 


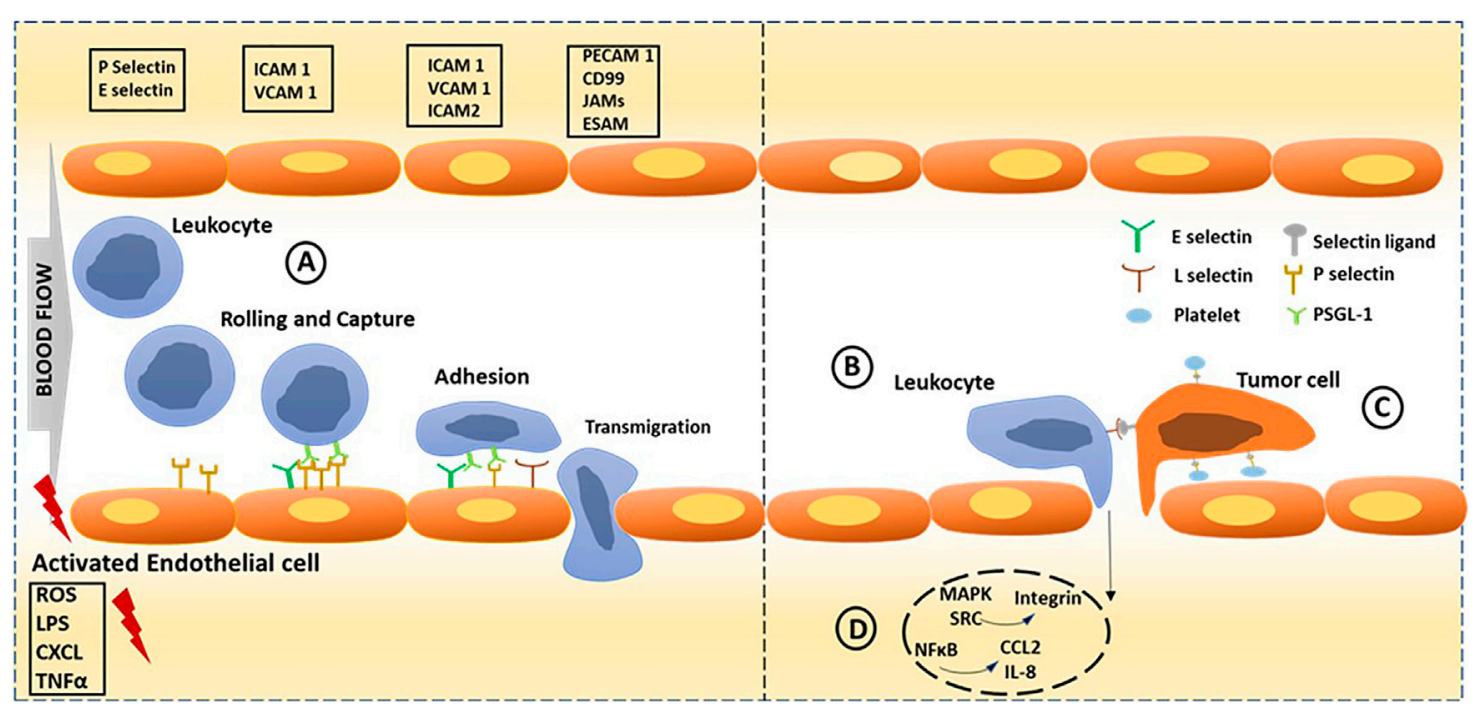

FIGURE 1 | Selectins dependent cell adhesion in normal condition and cancer progression. Endothelial activation is triggered through endogenous and exogenous stimuli from inflamed tissue. (A) Leukocyte adhesion cascade could be classified into three steps: rolling; capture, activation and arrest; which is mediated by selectins, chemokines and integrins respectively, progressing to transcellular migration. (B) Selectin-triggered endothelial activation leads to leukocyte-assisted tumor cell extravasation. Cytokines produced by the tumor cells lead to endothelial activation and leaky vasculature, resulting in leukocyte recruitment and their extravasation. (C) Tumor cell adhesion is promoted by platelets binding to endothelium and tumor cells. (D) Intracellular NFkB signalling initiated by selectin binding leading to inflammation through activation of MAPK, SRC pathways leading to activation of integrins and secretion of cytokines like CCL2, IL-8 and TNF- $\alpha$. Key molecules involved in each step is shown in boxes; PSGL1: P-selectin glycoprotein ligand 1; ICAM 1 and 2: Intercellular adhesion molecule 1 and 2; VCAM 1: Vascular cell adhesion molecule 1; PECAM 1: Platelet/endothelial cell adhesion molecule 1; JAM: junctional adhesion molecule; ESAM: endothelial cell-selective adhesion molecule; ROS: Reactive oxygen species; LPS: lipopolysaccharide; TNFa: tumor necrosis factor- $\alpha$; CXCL: Chemokine (C-X-C motif) ligand.

TABLE 1 | Class of nanoparticles used in drug delivery and imaging targeted towards selectins.

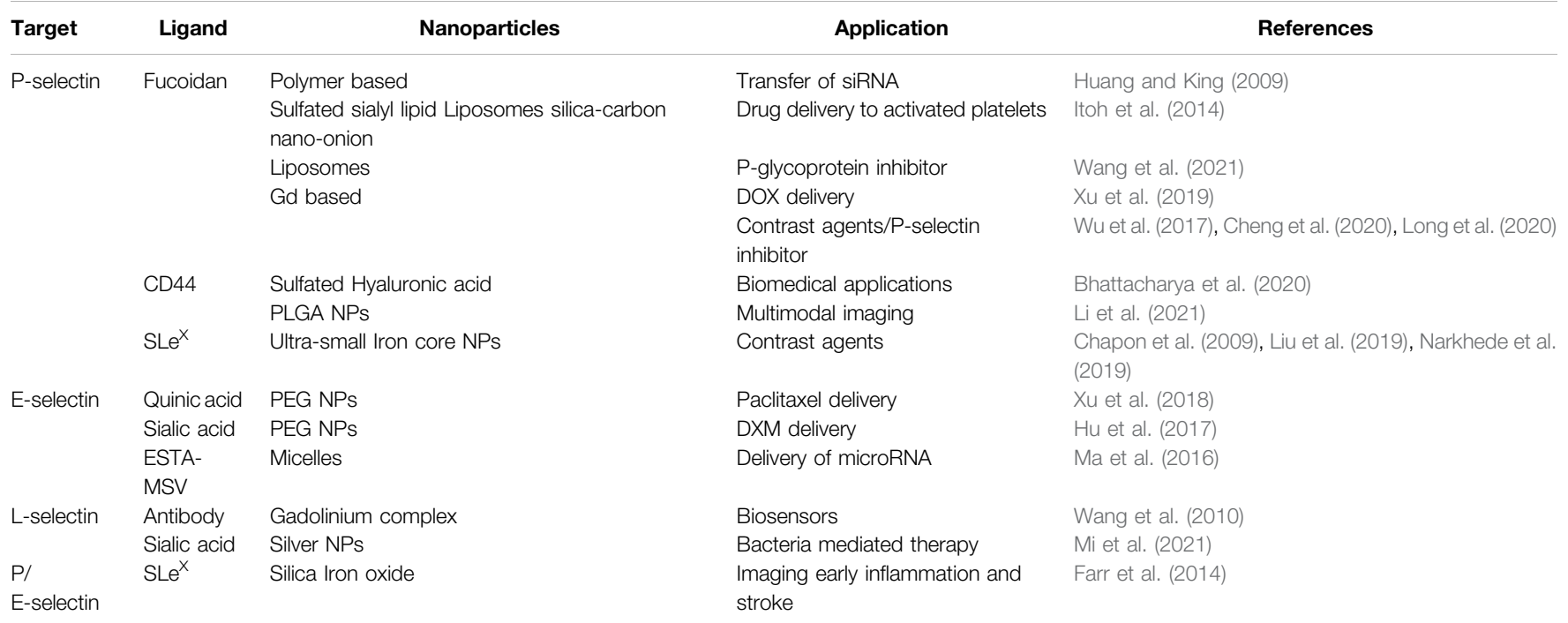

Abbreviations: DOX, Doxorubicin; DXM, Dexamethasone; ESTA-MSV, E-Selectin targeted multistage vector; Gd(III), Gadolinium; PLGA, Poly Lactic-co-Glycolic acid; PEG, Polyethylene glycol; SLe ${ }^{x}$, Sialyl-Lewis ${ }^{x}$.

chemokine (C-C motif) ligand 2 (CCL2), Interleukin-8 and Tumor necrosis factor- $\alpha$ (TNF $\alpha$ ) (Figure 1). It has been known since decades that selectins are expressed only at activated platelets and endothelial cells. The activated platelets undergo rearrangement of membrane glycoprotein that in turn is believed to cause rapid series of morphological and biochemical changes. Owing to the overexpression of selectins on activated platelets, covering the entire circulatory system from head to toe, they turned out to be a therapeutic target for several inflammatory diseases, thrombosis, and cardiac diseases 
(Ludwig et al., 2007). Furthermore, researchers proved that selectins may also promote cancer metastasis and tumor growth (Heinz and Lubor, 2010; McEver and Martin, 1984; Berman et al., 1986; Wu, 1996; Blann and Lip, 1997) (Figure 1), indicating that selectins have important functions besides merely cell adhesion. Therefore, selectin-binding ligandembedded nanostructures are an exciting tool for designing various biomaterials to target these inflammatory diseases and cardiovascular disorders, as well as cancer and metastasis. Many reviews have already reported the potential applications of selectin targeting in cancer biology and neurobiology. Herein, we specifically focus on multivalent probes such as nanoparticles, polymers and liposomes, and their inherent physical properties, making them an excellent candidate for studying selectin mediated interactions and potential applications.

\section{SELECTINS AND ITS LIGANDS}

Selectins are transmembrane glycoproteins that belong to the C-type lectin family since they exhibit $\mathrm{Ca}^{2+}$-dependent glycan binding. The selectin family is made up of P-, E-, and L-selectin with diverse functions and cell adhesion properties. The standard selectin structure contains a C-type lectin domain followed by an epidermal growth factor (EGF)-like domain and short sushi extracellular domains (McEver and Zhu, 2010). P-selectin (CD62P) and E-selectin (CD62E) are expressed on endothelial cells through the activation of pro-inflammatory cytokines, such as tumor necrosis factor (TNF)- $\alpha$, interleukin $-1 \beta$ (IL-1 $\beta$ ), and lipopolysaccharides (Tedder et al., 1995). Generally, E-selectin complements $\mathrm{P}$-selectin binding to recruit leukocytes to the inflammatory site (RP and C, 2010). P-selectin is selectively expressed on activated platelets (Bonfanti et al., 1989). It contributes to platelet adhesion to neutrophils, monocytes, and NK cells, and drives immune cells to injury sites (Chacko et al., 2011). By comparison, L-selectin is expressed on naïve T- and B-cells, myeloid cells, and leukocytes (Rosen, 2004). L-selectin mediates the recirculation of lymphoid cells from lymph nodes to blood circulation and vice versa (Miyasaka et al., 2016). Like the other two selectins, L-selectin also mediates leukocyte adhesion at inflammatory sites (Figure 1). The only difference between the L-selectin adhesion to $\mathrm{P}$ and E-selectin adhesion is that it can even occur under high blood flow-rate conditions. These proteins utilize P-selectin glycoprotein ligand-1 (PSGL-1 or CD162) as the primary ligand on leukocytes and hematopoietic stem cells to regulate physiological functions (Moore, 1998). PSGL-1 is a homodimer protein expressed on cell surfaces that undergoes posttranslational modification with sialyl Lewis ${ }^{\mathrm{x}}\left(\mathrm{SLe}^{\mathrm{x}}\right)$ glycan at $\mathrm{N}$-terminals and binds to the selectin carbohydrate-recognition domain (CRD) to regulate the tethering, rolling, and adhesion of leukocytes at the inflammatory site (McEver and Zhu, 2010). In addition to SLe ${ }^{\mathrm{x}}$ and $\mathrm{P}$ - and L-selectin, E-selectin did not show binding affinity to sulfated forms of SLe ${ }^{\mathrm{x}}$ and heparan sulfate/ heparin ligands (Brunk and Hammer, 1997). Additionally, several biomimetic analogs of $\mathrm{SLe}^{\mathrm{x}}$ and HS, such as quinic acid and fucoidan (Amoozgar et al., 2013), have been reported as potential ligands that target selectins. Furthermore, polypeptide sequences such as IELLQAR showed strong inhibition of sialyl Lewis ${ }^{\mathrm{X}}$ binding to E-selectin, as endothelial cell-leukocyte interaction and platelet-leukocyte interaction are involved in coagulation, inflammation, and metastasis (Chapon et al., 2009).

\section{SELECTINS AND DISEASES}

\section{Thrombosis and Thrombolysis}

Thrombosis is a blood clotting process in veins and arteries, preventing the blood flow in the circulation system and causing illnesses, including acute myocardial infarction, vein thrombosis, and ischemic stroke. Platelets play a fundamental role in preventing blood loss by activating coagulation factors and thereby inducing platelet hemostatic plug. At the injury site, the release of nitric oxide and prostaglandin $\mathrm{I}_{2}$ activate endothelial cells, which modulate the expression level of various cell-adhesion molecules, including $p$-selectin, to regulate platelet adhesion and leukocyte adhesion recruitment, fibrinolysis, inflammation. Hence P-selectin targeting and effective delivery of thrombolytic drug at the endothelial cell/ thrombus site could improve the efficacy of thrombolytics (Atherton and Born, 1972). Recently, the vast majority of P-selectin binding ligands scaffolds have been used in thrombolysis and imaging thrombosis.

\section{Endothelial Dysfunction}

Endothelial cells (ECs) play important roles in our lives, as they constitute a thick cell wall layer of arteries, veins, and capillaries. ECs regulate the transport of oxygen, nutrients, and immune cells across different body tissues and maintain blood pressure and blood circulation (Kasteren et al., 2009). Given their diverse biological functions, any inflammation in endothelial cells triggers cell dysfunction, resulting in several diseases. Some diseases, such as stroke, heart disease, chronic kidney failure, angiogenesis, and acute lung injury, are directly associated with vascular function, and other diseases, such as immune disorders, are indirectly associated with dysregulation of immune cell responses at the inflammatory site (Rajendran et al., 2013). Hence, early detection of inflammatory endothelial cells could provide valuable information about several diseases. Previously, it has been shown that E-selectin plays a role in endostatinmediated anti-angiogenesis (Yu et al., 2004), which promotes and/or inhibits blood vessel formation. Similarly, alveolarcapillary barriers are extensively damaged in acute lung injury (ALI) (Liu, 2019). Thus, abundantly expressed E-selectin on inflammatory endothelial cells acts as a potential therapeutic target for ALI. Furthermore, leukocyte-activated endothelial cell interaction is a hallmark of several neurological disorders, such as multiple sclerosis (MS) (Filippi et al., 2018) and ischemic stroke (Yilmaz and Granger, 2010). Consequently, early diagnosis of this activation is a highly desirable approach.

\section{Cancer}

Cancer cells overexpress selectin binding ligands, as well as selectins on their cell surfaces and promote cancer evasion. These substrates regulate tumor extravasation and support 


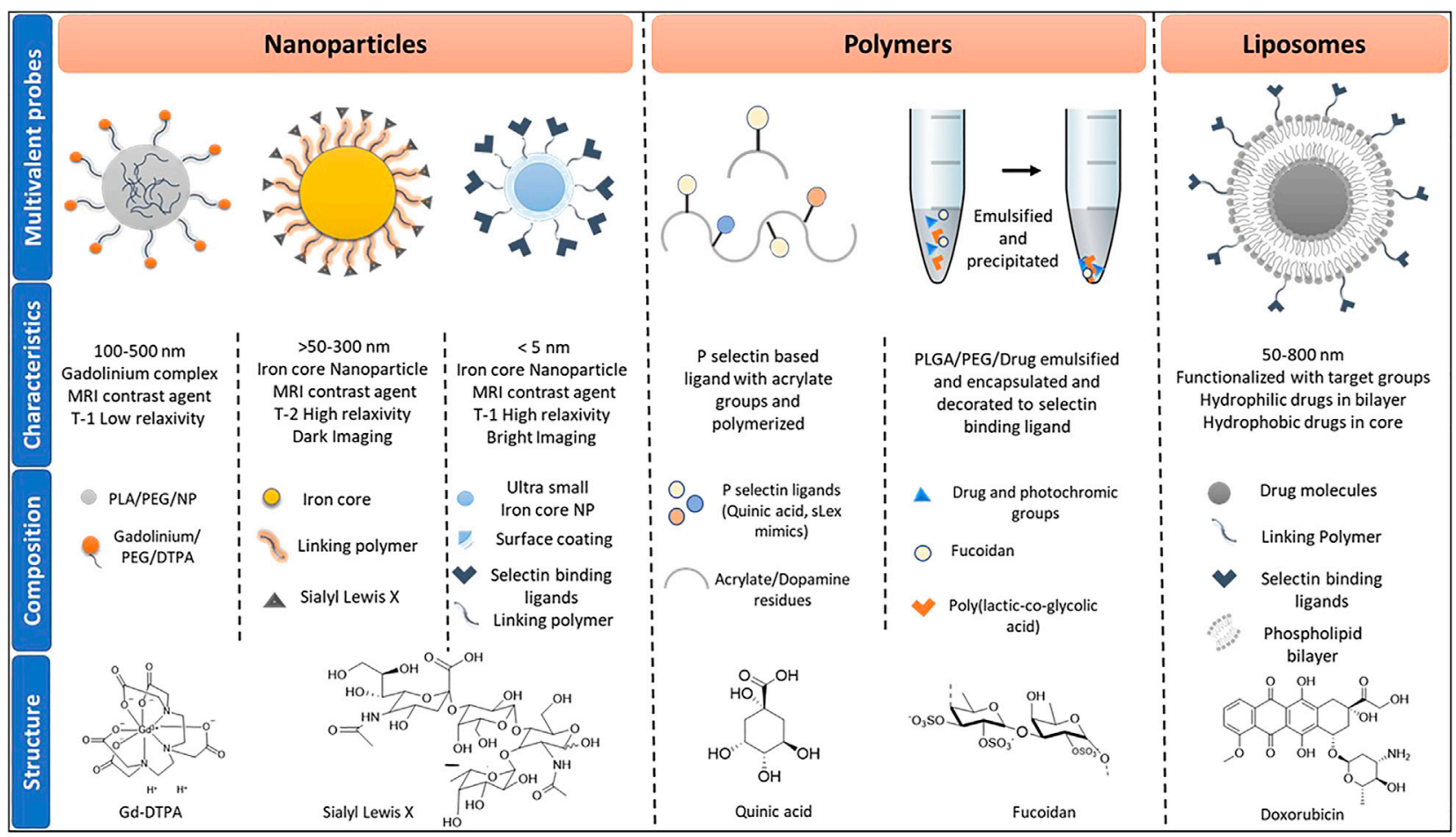

FIGURE 2 | Classes of multiprobes used in imaging and drug delivery, inclusive of their characteristics and composition. Abbreviations used are termed as follows, PLA: Polylactic acid; PEG: Polyethylene glycol; NP: nanoparticles: DTPA: Diethylenetriamine pentaacetate; sLex: Sialyl Lewis x; PLGA: Polylactic-co-glycolic acid.

tumor cells rolling on the activated endothelium. Recent immunohistochemistry studies with solid tumor samples revealed that $\mathrm{P}$-selectin expressed on most of the tumor types, including lung (19\%), ovarian (68\%), lymphoma (78\%), and breast (48\%) (Shamay et al., 2016). Further, radiation ionization of solid tumor and endothelial cells showed P-selectin translocation on cell membrane (Shamay et al., 2016). Elevated P-selectin has been found to mediate cancer cell adhesion on endothelial cells and platelets, resulting in thrombosis (Merten and Thiagarajan, 2004), cancer mortality (Borsig, 2018), and vasculature (Tedder et al., 1995). Therefore, selective targeting of $\mathrm{P}$-selectins and subsequent anticancer drug delivery could impact different aspects of tumor progression.

\section{MULTIVALENT PROBES}

Multivalent binding governs many biological interactions via the sum of cooperative interactions between ligand-receptors that increase binding avidity rather than affinity (Kiessling et al., 2008). In the last few decades, multivalent probes have shown great promise for gaining new insight into carbohydrate-mediated interactions (Marradi et al., 2013). Consequently, efforts have been made to capture the biological events regulated by selectin-mediated interaction using various multivalent probes. The most prevalent multivalent probes are nanoparticles, liposomes, and polymers (Figure 2).

\section{NANOPARTICLES}

Usually referred to the particles size of between 1 and $100 \mathrm{~nm}$ in diameter. At this tiny size, particles start behaving differently from that of bulk materials. Researchers have exploited the nanoparticles' inherent optical, electronic, magnetic properties to generate imaging and biomedical applications (Khan et al., 2019). Several types of nanoparticles have been synthesized to target selectin-mediated biological interaction (Table 1). Among them, iron oxide nanoparticles are the most predominant one, due to its inherent magnetic resonance imaging (MRI) contrast agent to develop the real-time imaging of selectin-mediated interactions (Stephen et al., 2011). These magnetic nanoparticles have several advantages over Gadolinium based contrast agent such as Gd-DTPA, which has a low T1 relaxivity (Cheng et al., 2020). The development of iron oxide nanoparticles-based MRI imaging displayed high T2 relaxivity, essential to visualize deep neural networks and tissues. Previously, Ben Davis et al. and Seeberger et al. employed sialyl lewisx conjugated silica iron oxide nanoparticles to study neural inflammation in the ischemic stroke mice model (Farr et al., 2014). MRI imaging of the brain demonstrated the feasibility of imaging endothelial activation in vivo after acute stroke ( $\mathrm{Lu}$ J. et al., 2021). However, the surgical procedure to develop a stroke model enhances P-selectin expression, and therefore the exact level of P-selectin expression is difficult to interpret. Furthermore, the T2 relaxivity of iron oxide nanoparticles that produce dark MRI images induces a high background interface. 
Hence, IONPs are taken off of the market and looking for an alternative NPs based contrast agent. Recently, ultrasmall iron oxide nanoparticles (USIONPs) of size $<4 \mathrm{~nm}$ were used as a positive T1 relaxivity MRI contrast agent, as they offer bright MRI imaging and high-resolution, resulting in easy detection of the target (Liu et al., 2019). Recently, Rao et al. reported the synthesis of quinic acid (QA)-coated USIONPs to target P-selectin overexpressed aggressive cancer cells (Narkhede et al., 2019). Working under the same notion, Liu et al. reported the synthesis of USIONPs-PEG-SLex nanoparticles to assess in vivo E-selectin expression level in nasopharyngeal carcinoma (NPC) metastasis (Liu et al., 2019). On considering of the inherent magnetic properties of USIONPs, this is a valuable tool for MRI imaging of ischemic stroke.

Bacterial mediated tumor therapy (BMTT) is an attractive target to cure cancer (Liu, 2019). However, neutrophil recruitment to the tumor site eliminates the bacteria and induces an immunological barrier for BMTT (Mi et al., 2021). As a result, neutrophil-depleting strategies have been employed to improve BMTT cancer therapy. Recently, Rong and co-worked synthesized sialic acid-coated silver nanoparticles to target L-selectin on neutrophils (Mi et al., 2021). These glyco-AgNPs showed neutrophilic depletion and increased salmonella bacteria's efficacy, which directly killed tumor cells and demonstrated superior therapeutic potentials (Mi et al., 2021). However, the pathogenicity of the bacteria can also have a large effect real-time application of BMTT.

\section{POLYMERS}

Polymers are powerful multivalent probes, as they offer a wide range of molecular weight, cheaper, biocompatible and readily scalable materials. There are two types of polymeric systems readily used in selectin-mediated studies. In the first set of selectin-targeted polymers, selectins specific ligands, such as quinic acid, sLex mimics, were functionalized on acrylate or dopamine residue and employed polymerization strategy to obtain desired polymeric nanoparticles (Amoozgar et al., 2013). However, the functional groups such as carboxylic acid and hydroxy groups quench the free radical polymerization, resulting in low efficacy and high polydispersity. Alternatively, polymeric nanoparticles were formulated by the emulsion-solvent evaporation method encapsulated with drug molecules and selectin binding ligands (Kona et al., 2012). This method offers several advantages including the mild condition to formulate biocompatible and biodegradable nanoparticles without compromising the activity of drug. Moreover, the nanoparticles prepared by this method offer control delivery of drug molecules. Nguyen and co-workers reported the synthesis of drug (dexamethasone) and fluorescent tag (6coumarin dye) and glycoprotein Ib (GPIb; p-selectin binding ligand)-loaded poly(lactide-co-glycolide) nanoparticles to target P-selectin overexpressed endothelial cells and drug delivery (Inyang et al., 2020). Similarly, Ran and co-workers synthesized polymeric nanoparticles composed of photothermal agents such as nanocarbons, doxorubicin and perfluropentane encapsulated polymeric nanoparticles coated with platelet membrane (Li et al., 2021). These multicomponent nanoplatelets were used to demonstrate breast cancer theranostic. Mizrachi et al. synthesized fucoidanbased fluorescent nanoparticles loaded with BYL719, a $\mathrm{PI} 3 \mathrm{Ka}$ inhibitor to target squamous cell carcinoma (Mizrachi et al., 2017). Lee et al. synthesized fucoidandoxorubicin nanoparticles to target P-selectin overexpressed cancer cells. While, Chuang et al. synthesized fucoidan-coated polypyrrole nanoparticles to target cancer cells and generate photothermal treatment of tumors (Lu K.-Y. et al., 2021). Overall, these results all show the huge potential of polymer-based nanoparticles in real-time applications of cancer therapy.

\section{LIPOSOMES}

Liposomes are one of the attractive nanocarriers for controlled cargo delivery, composed of lipid bilayers in a discrete aqueous environment. They can host both hydrophilic drug molecules in the aqueous centre and hydrophobic molecules between the lipid bilayers and display a large surface area to functionalize biological ligands, including carbohydrates, peptides, and proteins (Sercombe et al., 2015). Liposomes are extensively used to design selectin-mediated drug delivery and imaging system to target various diseases (Guimarães et al., 2021). Li et al. (2020) synthesized sialic acid-modified doxorubicin based liposomes to target and kill peripheral blood neutrophils via sialic acid-L-selectin interaction to reduce the accumulation of neutrophile at the rheumatoid arthritis (RA) disease site. Similarly, Matsumura et al. synthesized sialyl lewis X-modified doxo-liposomes to target injured vessel walls to prevent stenosis after angioplasty (Tsuruta et al., 2009). While, Zalipsky et al. reported silyl lewis X liposomes to develop antiadhesion molecules (DeFrees et al., 1996). Azab et al., prepared bone marrow microenvironment destructing inhibitor modified $\mathrm{P}$-selectin glycoprotein ligand-1 conjugated liposome to target multiple myeloma-associated endothelium (Azab et al., 2012). However, single-ligands often fail to target the dynamic microenvironment of the tumor, particularly metastasis cancer cells. To improve the accuracy in targeting metastasis cancer cells, multi-ligand embedded liposomal nanoparticles have been synthesized. Here, $\mathrm{P}$-selectin-specific ligands, integrin-targeting peptides, fibronectin targeting peptides, and epidermal growth factor receptor (EGFR) targeting peptides were assembled on a single liposome to target more than one receptor overexpressed on cancer cells (Peiris et al., 2018). By employing such multiligand strategies, highly sensitive and precise imaging of earlystage cancer cells metastasis was achieved (Doolittle et al., 2015; Peiris et al., 2018). Overall, selectin-ligands conjugated liposomes have shown enormous potential in theranostics. However, the poor cost-effectiveness of liposome-based drug-delivery limited its clinical translation. 


\section{FUTURE DIRECTION AND SUMMARY}

This mini-review highlights the impressive work carried out by different research groups functionalizing the nanoprobes with selectins targeting ligands and their potential theranostic applications. Nanoprobes' distinct magnetic, fluorescent, and drug carrier ability was used to probe selective targeting and drug delivery to treat cancer, inflammatory diseases, and thrombosis. However, the main challenge is the weak and reversible binding affinity of native ligands such as SLe ${ }^{\mathrm{x}}$ and SLe ${ }^{\mathrm{a}}$ to selectins (Brunk and Hammer, 1997). Hence, there is a lot of scope to design and identify synthetic ligands from heparan sulfate to improve selectins' binding affinity and specificity. Furthermore, selectin nanoprobes targeting tumor microenvironment and brain strokes must pass through specific barriers, including blood brain barrier (BBB) and extracellular matrix/stromal cell barriers. These barriers drastically influence the number of nanoparticles delivered to the active sites. Hence there is a great deal of interest in modifying nanoprobes to improve their efficacy.

In summary, leukocyte-activated endothelial and plateletendothelial cell interaction plays a critical role in various

\section{REFERENCES}

Amoozgar, Z., Park, J., Lin, Q., Weidle, J. H., and Yeo, Y. (2013). Development of Quinic Acid-Conjugated Nanoparticles as a Drug Carrier to Solid Tumors. Biomacromolecules 14, 2389-2395. doi:10.1021/bm400512g

Atherton, A., and Born, G. V. R. (1972). Quantitative Investigations of the Adhesiveness of Circulating Polymorphonuclear Leucocytes to Blood Vessel walls. J. Physiol. 222, 447-474. doi:10.1113/ JPHYSIOL.1972.SP009808

Azab, A. K., Quang, P., Azab, F., Pitsillides, C., Thompson, B., Chonghaile, T., et al. (2012). P-selectin Glycoprotein Ligand Regulates the Interaction of Multiple Myeloma Cells with the Bone Marrow Microenvironment. Blood 119, 1468-1478. doi:10.1182/BLOOD-2011-07-368050

Berman, C. L., Yeo, E. L., Wencel-Drake, J. D., Furie, B. C., Ginsberg, M. H., and Furie, B. (1986). A Platelet Alpha Granule Membrane Protein that Is Associated with the Plasma Membrane after Activation. Characterization and Subcellular Localization of Platelet Activation-dependent Granule-External Membrane Protein. J. Clin. Invest. 78, 130-137. doi:10.1172/JCI112542

Bhattacharya, D. S., Svechkarev, D., Bapat, A., Patil, P., Hollingsworth, M. A., and Mohs, A. M. (2020). Sulfation Modulates the Targeting Properties of Hyaluronic Acid to P-Selectin and CD44. ACS Biomater. Sci. Eng. 6, 3585-3598. doi:10.1021/acsbiomaterials.0c00115

Blann, A. D., and Lip, G. Y. H. (1997). Hypothesis: Is Soluble P-Selectin a New Marker of Platelet Activation? Atherosclerosis 128, 135-138. doi:10.1016/S00219150(96)05980-1

Bonfanti, R., Furie, B., Furie, B., and Wagner, D. (1989). PADGEM (GMP140) Is a Component of Weibel-Palade Bodies of Human Endothelial Cells. Blood 73, 1109-1112. doi:10.1182/BLOOD.V73.5.1109.110910.1182/ blood.v73.5.1109.bloodjournal7351109

Borsig, L. (2018). Selectins in Cancer Immunity. Glycobiology 28, 648-655. doi:10.1093/GLYCOB/CWX105

Brunk, D. K., and Hammer, D. A. (1997). Quantifying Rolling Adhesion with a Cell-free Assay: E-Selectin and its Carbohydrate Ligands. Biophysical J. 72, 2820-2833. doi:10.1016/S0006-3495(97)78924-0

Chacko, A.-M., Hood, E. D., Zern, B. J., and Muzykantov, V. R. (2011). Targeted Nanocarriers for Imaging and Therapy of Vascular Inflammation. Curr. Opin. Colloid Interf. Sci. 16, 215-227. doi:10.1016/j.cocis.2011.01.008 human disorders, including thrombosis, stroke, heart disease, kidney failure, tumor growth metastasis. Hence selectin-binding ligand conjugated nanoprobes serve as a potential marker for the early detection of these diseases and their prevention and more effective treatment. SLe ${ }^{\mathrm{X}}$, fucoidan, quinic acid and selectinbinding peptides have been used for quantitative targeting selectin-mediated interactions. In addition, $\mathrm{SLe}^{\mathrm{X}}$-Iron nanoparticles have been extensively exploited for MRI imaging of brain stroke and cancer metastasis.

\section{AUTHOR CONTRIBUTIONS}

DG and PJ conceptualize the approach. All authors wrote the article.

\section{ACKNOWLEDGMENTS}

RK gratefully acknowledges financial support from the IISER, Pune, DBT (grant nos. BT/PR34475/MED/15/210/2020), STARS/APR2019/CS/426/FS and SERB/F/9228/2019-2020 for financial support.

Chapon, C., Franconi, F., Lacoeuille, F., Hindré, F., Saulnier, P., Benoit, J.-P., et al. (2009). Imaging E-Selectin Expression Following Traumatic Brain Injury in the Rat Using a Targeted USPIO Contrast Agent. Magn. Reson. Mater. Phy 22, 167-174. doi:10.1007/s10334-008-0161-Z

Cheng, T.-M., Li, R., Kao, Y.-C. J., Hsu, C.-H., Chu, H.-L., Lu, K.-Y., et al. (2020). Synthesis and Characterization of Gd-DTPA/fucoidan/peptide Complex Nanoparticle and In Vitro Magnetic Resonance Imaging of Inflamed Endothelial Cells. Mater. Sci. Eng. C 114, 111064. doi:10.1016/ j.msec.2020.111064

Chiara, C., Virgilio, E., and Giovanni, de. G. (1999). P-selectin-beta 2-integrin Cross-Talk: a Molecular Mechanism for Polymorphonuclear Leukocyte Recruitment at the Site of Vascular Damage. Thromb. Haemost. 82, 787-793. Available at: https://pubmed.ncbi.nlm.nih.gov/10605783/(Accessed September 8, 2021).

De Gaetano, G., Donati, M. B., and Cerletti, C. (2003). Prevention of Thrombosis and Vascular Inflammation: Benefits and Limitations of Selective or Combined COX-1, COX-2 and 5-LOX Inhibitors. Trends Pharmacol. Sci. 24, 245-252. doi:10.1016/S0165-6147(03)00077-4

DeFrees, S. A., Phillips, L., Guo, L., and Zalipsky, S. (1996). Sialyl Lewis X Liposomes as a Multivalent Ligand and Inhibitor of E-Selectin Mediated Cellular Adhesion. J. Am. Chem. Soc. 118, 6101-6104. doi:10.1021/ja954122g

Doolittle, E., Peiris, P. M., Doron, G., Goldberg, A., Tucci, S., Rao, S., et al. (2015). Spatiotemporal Targeting of a Dual-Ligand Nanoparticle to Cancer Metastasis. ACS Nano 9, 8012-8021. doi:10.1021/acsnano.5b01552

Farr, T. D., Lai, C.-H., Grünstein, D., Orts-Gil, G., Wang, C.-C., Boehm-Sturm, P., et al. (2014). Imaging Early Endothelial Inflammation Following Stroke by Core Shell Silica Superparamagnetic Glyconanoparticles that Target Selectin. Nano Lett. 14, 2130-2134. doi:10.1021/nl500388h

Filippi, M., Bar-Or, A., Piehl, F., Preziosa, P., Solari, A., Vukusic, S., et al. (20182018). Multiple Sclerosis. Nat. Rev. Dis. Primers 4, 1-27. doi:10.1038/ s41572-018-0041-4

Guimarães, D., Cavaco-Paulo, A., and Nogueira, E. (2021). Design of Liposomes as Drug Delivery System for Therapeutic Applications. Int. J. Pharmaceutics 601, 120571. doi:10.1016/J.IJPHARM.2021.120571

Heinz, L., and Lubor, B. (2010). Selectins Promote Tumor Metastasis. Semin. Cancer Biol. 20, 169-177. doi:10.1016/J.SEMCANCER.2010.04.005

Hu, J.-B., Song, G.-L., Liu, D., Li, S.-J., Wu, J.-H., Kang, X.-Q., et al. (2017). Sialic Acid-Modified Solid Lipid Nanoparticles as Vascular Endothelium-Targeting 
Carriers for Ischemia-Reperfusion-Induced Acute Renal Injury. Drug Deliv. 24, 1856-1867. doi:10.1080/10717544.2017.1410258

Huang, Z., and King, M. R. (2009). An Immobilized Nanoparticle-Based Platform for Efficient Gene Knockdown of Targeted Cells in the Circulation. Gene Ther. 16, 1271-1282. doi:10.1038/gt.2009.76

Inyang, E., Kuriakose, A. E., Chen, B., Nguyen, K. T., and Cho, M. (2020). Engineering Delivery of Nonbiologics Using Poly(lactic-Co-Glycolic Acid) Nanoparticles for Repair of Disrupted Brain Endothelium. ACS Omega 5, 14730-14740. doi:10.1021/acsomega.0c01517

Itoh, S., Kawano, K., Takeshita, K., Maitani, Y., and Tsuji, T. (2014). Development of Liposomal Nanoconstructs Targeting P-Selectin (CD62P)-Expressing Cells by Using a Sulfated Derivative of Sialic Acid. Pharm. Res. 31, 2868-2875. doi:10.1007/s11095-014-1383-6

Khan, I., Saeed, K., and Khan, I. (2019). Nanoparticles: Properties, Applications and Toxicities. Arabian J. ChemistryJ. Chem. 12, 908-931. doi:10.1016/ J.ARABJC.2017.05.011

Kiessling, L. L., Young, T., Gruber, T. D., and Mortell, K. H. (2008). Multivalency in Protein-Carbohydrate Recognition. Glycoscience 45, 2483-2523. doi:10.1007/ 978-3-540-30429-6_64

Kona, S., Dong, J.-F., Liu, Y., Tan, J., and Nguyen, K. T. (2012). Biodegradable Nanoparticles Mimicking Platelet Binding as a Targeted and Controlled Drug Delivery System. Int. J. Pharmaceutics 423, 516-524. doi:10.1016/ j.ijpharm.2011.11.043

Li, C., Qiu, Q., Liu, M., Liu, X., Hu, L., Luo, X., et al. (2020). Sialic Acid-Conjugate Modified Liposomes Targeting Neutrophils for Improved Tumour Therapy. Biomater. Sci. 8, 2189-2201. doi:10.1039/c9bm01732a

Li, L., Fu, J., Wang, X., Chen, Q., Zhang, W., Cao, Y., et al. (2021). Biomimetic "Nanoplatelets" as a Targeted Drug Delivery Platform for Breast Cancer Theranostics. ACS Appl. Mater. Inter. 13, 3605-3621. doi:10.1021/acsami.0c19259

Liu, D. (20192019). CAR-T "the Living Drugs", Immune Checkpoint Inhibitors, and Precision Medicine: a new era of Cancer Therapy. J. Hematol. Oncol. 12, 1-5. doi:10.1186/S13045-019-0819-1

Liu, L., Liu, L., Li, Y., Huang, X., Gu, D., Wei, B., et al. (2019). Ultrasmall Superparamagnetic Nanoparticles Targeting E-Selectin: Synthesis and Effects in Mice In Vitro and In Vivo. Ijn 14, 4517-4528. doi:10.2147/IJN.S199571

Long, L., Deng, L., Wang, L., Wen, S., Luo, L., Liang, L., et al. (2020). P-SelectinBased Dual-Model Nanoprobe Used for the Specific and Rapid Visualization of Early Detection toward Severe Acute Pancreatitis In Vivo. ACS Biomater. Sci. Eng. 6, 5857-5865. doi:10.1021/acsbiomaterials.0c00596

Lu, J., Mei, Q., Hou, X., Manaenko, A., Zhou, L., Liebeskind, D. S., et al. (2021a). Imaging Acute Stroke: From One-Size-Fit-All to Biomarkers. Front. Neurol. 12, 1276. doi:10.3389/FNEUR.2021.697779

Lu, K.-Y., Jheng, P.-R., Lu, L.-S., Rethi, L., Mi, F.-L., and Chuang, E.-Y. (2021b). Enhanced Anticancer Effect of ROS-Boosted Photothermal Therapy by Using Fucoidan-Coated Polypyrrole Nanoparticles. Int. J. Biol. Macromolecules 166, 98-107. doi:10.1016/j.ijbiomac.2020.10.091

Ludwig, R. J., Schön, M. P., and Boehncke, W.-H. (2007). P-selectin. Expert Opin. Ther. Targets 11, 1103-1117. doi:10.1517/14728222.11.8.1103

Ma, S., Tian, X. Y., Zhang, Y., Mu, C., Shen, H., Bismuth, J., et al. (2016). E-selectintargeting Delivery of microRNAs by Microparticles Ameliorates Endothelial Inflammation and Atherosclerosis. Sci. Rep. 6, 1-11. doi:10.1038/srep22910

Marradi, M., Chiodo, F., García, I., and Penadés, S. (2013). Glyconanoparticles as Multifunctional and Multimodal Carbohydrate Systems. Chem. Soc. Rev. 42, 4728-4745. doi:10.1039/C2CS35420A

McEver, R. P., and Martin, M. N. (1984). A Monoclonal Antibody to a Membrane Glycoprotein Binds Only to Activated Platelets. J. Biol. Chem. 259, 9799-9804. doi:10.1016/S0021-9258(17)42770-0

McEver, R. P., and Zhu, C. (2010). Rolling Cell Adhesion. Annu. Rev. Cel Dev. Biol. 26, 363-396. doi:10.1146/ANNUREV.CELLBIO.042308.113238

Merten, M., and Thiagarajan, P. (2004). P-selectin in Arterial Thrombosis. Z. Kardiol. 93, 855-863. doi:10.1007/S00392-004-0146-5

Mi, Z., Guo, L., Liu, P., Qi, Y., Feng, Z., Liu, J., et al. (2021). "trojan Horse" Salmonella Enabling Tumor Homing of Silver Nanoparticles via Neutrophil Infiltration for Synergistic Tumor Therapy and Enhanced Biosafety. Nano Lett. 21, 414-423. doi:10.1021/acs.nanolett.0c03811

Miyasaka, M., Hata, E., Tohya, K., and Hayasaka, H. (2016). Lymphocyte Recirculation. Encycl. Immunobiol. 3, 486-492. doi:10.1016/B978-0-12374279-7.07013-2
Mizrachi, A., Shamay, Y., Shah, J., Brook, S., Soong, J., Rajasekhar, V. K., et al. (2017). Tumour-specific PI3K Inhibition via Nanoparticle-Targeted Delivery in Head and Neck Squamous Cell Carcinoma. Nat. Commun. 8. doi:10.1038/ ncomms 14292

Moore, K. L. (1998). Structure and Function of P-Selectin Glycoprotein Ligand-1. Leuk. Lymphoma 29, 1-15. doi:10.3109/10428199809058377

Narkhede, A. A., Sherwood, J. A., Antone, A., Coogan, K. R., Bolding, M. S., Deb, S., et al. (2019). Role of Surface Chemistry in Mediating the Uptake of Ultrasmall Iron Oxide Nanoparticles by Cancer Cells. ACS Appl. Mater. Inter. 11, 17157-17166. doi:10.1021/acsami.9b00606

Pachynski, R. K., Wu, S. W., Gunn, M. D., and Erle, D. J. (1998). Secondary Lymphoid-Tissue Chemokine (SLC) Stimulates Integrin Alpha 4 Beta 7mediated Adhesion of Lymphocytes to Mucosal Addressin Cell Adhesion Molecule-1 (MAdCAM-1) under Flow. J. Immunol. 161, 952-956.

Peiris, P. M., He, F., Covarrubias, G., Raghunathan, S., Turan, O., Lorkowski, M., et al. (2018). Precise Targeting of Cancer Metastasis Using Multi-Ligand Nanoparticles Incorporating Four Different Ligands. Nanoscale 10, 6861-6871. doi:10.1039/c8nr02513d

Rajendran, P., Rengarajan, T., Thangavel, J., Nishigaki, Y., Sakthisekaran, D., Sethi, G., et al. (2013). The Vascular Endothelium and Human Diseases. Int. J. Biol. Sci. 9, 1057-1069. doi:10.7150/ijbs.7502

Rosen, S. D. (2004). Ligands for L-Selectin: Homing, Inflammation, and beyond. Annu. Rev. Immunol. 22, 129-156. doi:10.1146/ annurev.immunol.21.090501.080131

Sercombe, L., Veerati, T., Moheimani, F., Wu, S. Y., Sood, A. K., and Hua, S. (2015). Advances and Challenges of Liposome Assisted Drug Delivery. Front. Pharmacol. 6, 286. doi:10.3389/FPHAR.2015.00286

Shamay, Y., Elkabets, M., Li, H., Shah, J., Brook, S., Wang, F., et al. (2016). P-selectin Is a Nanotherapeutic Delivery Target in the Tumor Microenvironment. Sci. Transl. Med. 8. doi:10.1126/scitranslmed.aaf7374

Stephen, Z. R., Kievit, F. M., and Zhang, M. (2011). Magnetite Nanoparticles for Medical MR Imaging. Mater. Today 14, 330-338. doi:10.1016/S1369-7021(11) 70163-8

Tedder, T. F., Steeber, D. A., Chen, A., and Engel, P. (1995). The Selecting: Vascular Adhesion Molecules. FASEB j. 9, 866-873. doi:10.1096/fasebj.9.10.7542213

Tsuruta, W., Tsurushima, H., Yamamoto, T., Suzuki, K., Yamazaki, N., and Matsumura, A. (2009). Application of Liposomes Incorporating Doxorubicin with Sialyl Lewis X to Prevent Stenosis after Rat Carotid Artery Injury. Biomaterials 30, 118-125. doi:10.1016/ J.BIOMATERIALS.2008.09.009

van Kasteren, S. I., Campbell, S. J., Serres, S., Anthony, D. C., Sibson, N. R., and Davis, B. G. (2009). Glyconanoparticles Allow Pre-symptomatic In Vivo Imaging of Brain Disease. Proc. Natl. Acad. Sci. 106, 18-23. doi:10.1073/ PNAS.0806787106

Wagner, D. D., Olmsted, J. B., and Marder, V. J. (1982). Immunolocalization of von Willebrand protein in Weibel-Palade bodies of human endothelial cells. J. Cel Biol. 95, 355-360. doi:10.1083/JCB.95.1.355

Wang, H., Liang, Y., Yin, Y., Zhang, J., Su, W., White, A. M., et al. (2021). Carbon Nano-Onion-Mediated Dual Targeting of P-Selectin and P-Glycoprotein to Overcome Cancer Drug Resistance. Nat. Commun. 12, 1-14. doi:10.1038/ s41467-020-20588-0

Wang, X.-F., Jin, P.-P., Tong Zhou, T., Zhao, Y.-P., Ding, Q.-L., Wang, D.-B., et al. (2010). MR Molecular Imaging of Thrombus: Development and Application of a Gd-Based Novel Contrast Agent Targeting to P-Selectin. Clin. Appl. Thromb. Hemost. 16, 177-183. doi:10.1177/1076029608330470

Wu, J., Zhu, H., Zhao, M., Wang, Y., Yang, G., Wang, Y., et al. (2017). IQCA-TASS: a Nano-Scaled P-Selectin Inhibitor Capable of Targeting Thrombus and Releasing IQCA/TARGD(S)S In Vivo. J. Mater. Chem. B 5, 917-927. doi:10.1039/c6tb02705a

Wu, K. K. (1996). Platelet Activation Mechanisms and Markers in Arterial Thrombosis. J. Intern. Med. 239, 17-34. doi:10.1046/J.13652796.1996.331661000.X

Xu, J., Cheng, X., Tan, L., Fu, C., Ahmed, M., Tian, J., et al. (2019). Microwave Responsive Nanoplatform via P-Selectin Mediated Drug Delivery for Treatment of Hepatocellular Carcinoma with Distant Metastasis. Nano Lett. 19, 2914-2927. doi:10.1021/acs.nanolett.8b05202

Xu, J., Lee, S. S. Y., Seo, H., Pang, L., Jun, Y., Zhang, R. Y., et al. (2018). Quinic AcidConjugated Nanoparticles Enhance Drug Delivery to Solid Tumors via 
Interactions with Endothelial Selectins. Small 14, 1803601-1803616. doi:10.1002/smll.201803601

Yilmaz, G., and Granger, D. N. (2010). Leukocyte Recruitment and Ischemic Brain Injury. Neuromol Med. 12, 193-204. doi:10.1007/S12017-0098074-1

Yu, Y., Moulton, K. S., Khan, M. K., Vineberg, S., Boye, E., Davis, V. M., et al. (2004). E-selectin Is Required for the Antiangiogenic Activity of Endostatin. Proc. Natl. Acad. Sci. 101, 8005-8010. doi:10.1073/ PNAS.0402551101

Conflict of Interest: The authors declare that the research was conducted in the absence of any commercial or financial relationships that could be construed as a potential conflict of interest.
Publisher's Note: All claims expressed in this article are solely those of the authors and do not necessarily represent those of their affiliated organizations, or those of the publisher, the editors and the reviewers. Any product that may be evaluated in this article, or claim that may be made by its manufacturer, is not guaranteed or endorsed by the publisher.

Copyright $\odot 2021$ Ganesh, Jain, Shanthamurthy, Toraskar and Kikkeri. This is an open-access article distributed under the terms of the Creative Commons Attribution License (CC BY). The use, distribution or reproduction in other forums is permitted, provided the original author $(s)$ and the copyright owner(s) are credited and that the original publication in this journal is cited, in accordance with accepted academic practice. No use, distribution or reproduction is permitted which does not comply with these terms. 